\title{
Linear analysis of complete structures \\ by computers
}

\section{K. I. MAJID \& M. WILLIAMSON}

\section{Professor F. Sawko and Mr R. J. Cope, University of Liverpool}

The Authors are to be congratulated on producing a comprehensive computer program for the solution of three dimensional structures based on the stiffness approach. The Paper was of great interest to us since, over the last four years, we have been concerned with the development of automatic computer programs for the analysis of two and three dimensional skeletal and plate structures. Unlike the Authors, however, we found it more profitable to concentrate our attention on the development of practical methods of analysis to specific types of structure in preference to producing an all embracing program.

42. This course of action was based upon our experience that solutions to three dimensional assemblages, required for the analysis of actual structures, could not economically be obtained with the present generation of computers. This finding seems to be supported by the Authors themselves in $\S 38$. Considerable storage space and computer times are quoted for the analysis of what is basically a very elementary type of structure and it is easy to see that the analysis of real engineering structures would be outside the capacity of even the Atlas computer.

43. Because of these conclusions the Authors' developments were mainly concerned with two dimensional systems, where the number of variables is very much smaller than with corresponding three dimensional structures. Occasionally, however, it is essential to investigate three dimensional stress distributions in structures which do not lend themselves to two dimensional idealization. An example of this is a spine beam bridge of the type shown in Fig. 13. To analyse structures of this

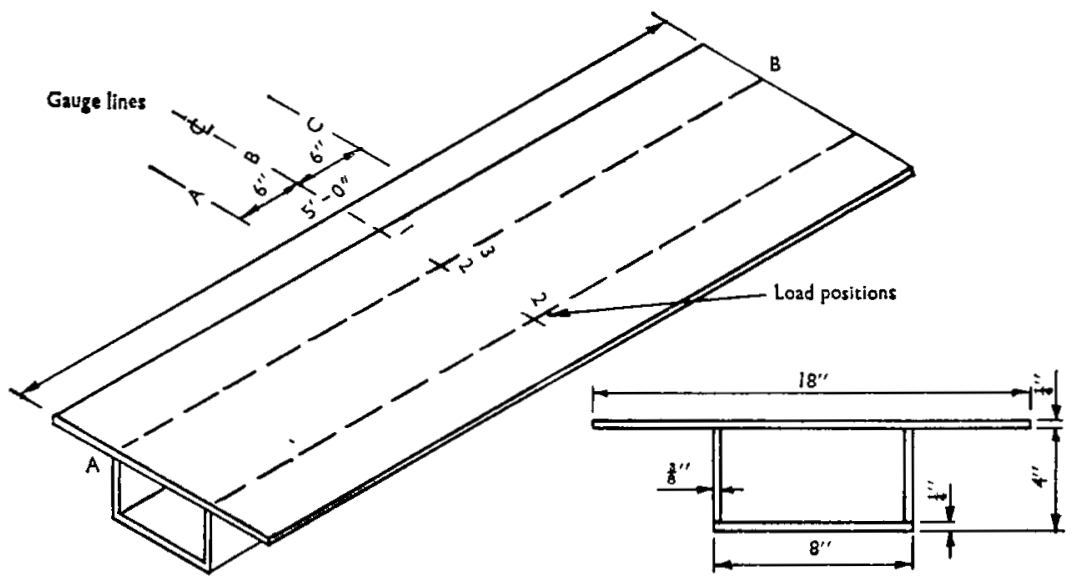

Fig. 13. Details of model spine beam bridge

Paper published: Proc. Instn civ. Engrs, 1967, 38 (Oct.) 247-266. 


\section{DISCUSSION}

type a three dimensional finite element program using rectangular elements capable of resisting direct and transverse stresses was developed. This lincar type of structural system lends itself to a considerable amount of optimization in solution techniques and a practical program was developed for the KDF9 computer. To reduce storage requirements and improve efficiency this utilized the well known stiffness matrix properties such as symmetry and the banding of terms about the leading diagonal. Facilities, in the form of magnetic tapes, were also incorporated to store intermediate results.

44. During the development of this program the difficulties with in-plane moments referred to in $\$ \$ 8$ and 15 were experienced and we were most interested in the Authors' approach. The difficulties arise at the junctions of non-coplanar elements, because in-plane stiffnesses are defined without recourse to in-plane rotations at the nodes of connecting elements. This difficulty is minimal for assemblages which resist only membrane stresses and solutions to such assemblages converge to the exact elasticity values with decreasing mesh size. It would seem, therefore, that the provision of additional prismatic clements, as advocated by the Authors, would lead to an over stiff structure.

45. Our approach consisted of allowing free rotations at the nodes along the junctions of non-coplanar elements. This line of action was thought to be on the conservative side as it was felt that the slight reduction in stiffness would lead to the prediction of larger stresses. The structure shown in Fig. 13 was analysed by our program and the nodal slopes along the slab-web junction are shown in Fig. 14. It is

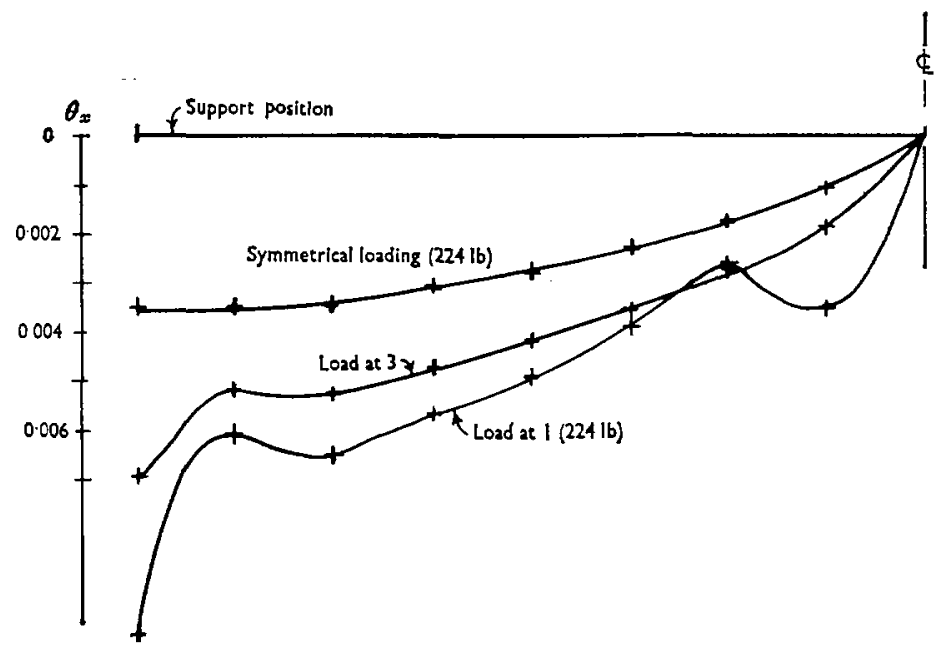

Fig. 14. Nodal slope along mesh line $A B$

immediately apparent that the variation of nodal slope under symmetrical loading and under loading over the webs produces a relatively smooth curve but that under eccentric loading there are considerable local nuctuations. In view of these observations it would seem that neither approach is entirely satisfactory and a new one might be required. It seems reasonable to suggest that for finite element assemblages it may be possible to derive a stiffness matrix using deflexion functions that are compatible with the normal displacement of the flexural function. This would involve defining a cubic displacement variation along mesh lines and could be achieved by adding two 
slopes at each node to the in-plane nodal displacements. We would be very interested to have the Authors' views on these comments.

46. The analysis of the model of Fig. 13 was carried out by dividing each plate member into a $2 \times 8$ mesh for one half of the symmetrical structure. The degree of accuracy obtained for deflexions and stresses is indicated in Figs 15, 16, 17 and 18. The computer solution is remarkably accurate considering the very coarse mesh that was used. The three dimensional system in the form of a spine beam bridge does lend itself to an economic solution but we would hesitate before advocating the

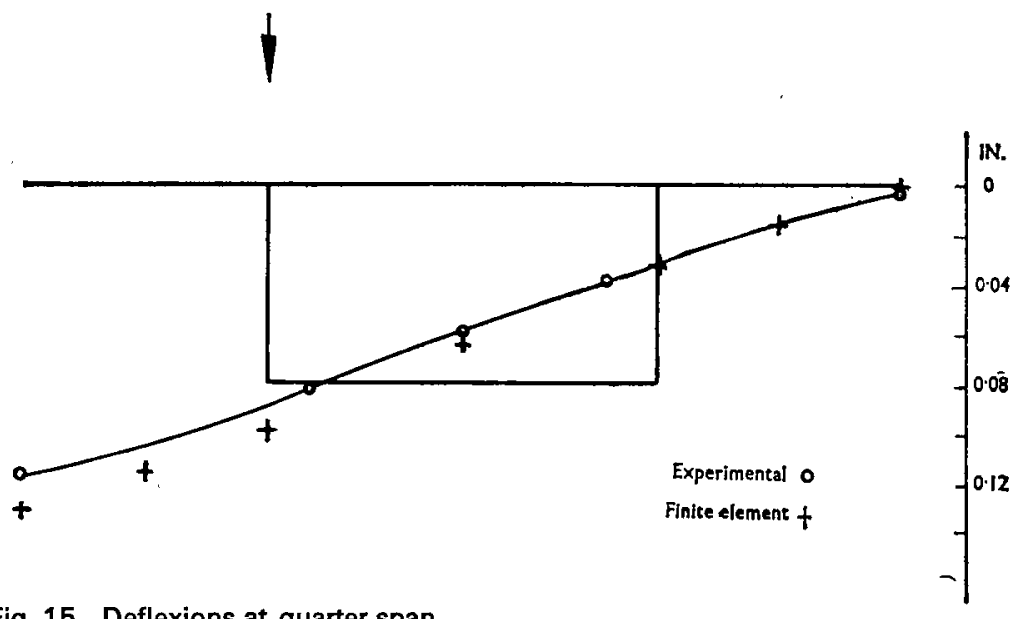

Fig. 15. Deflexions at quarter span

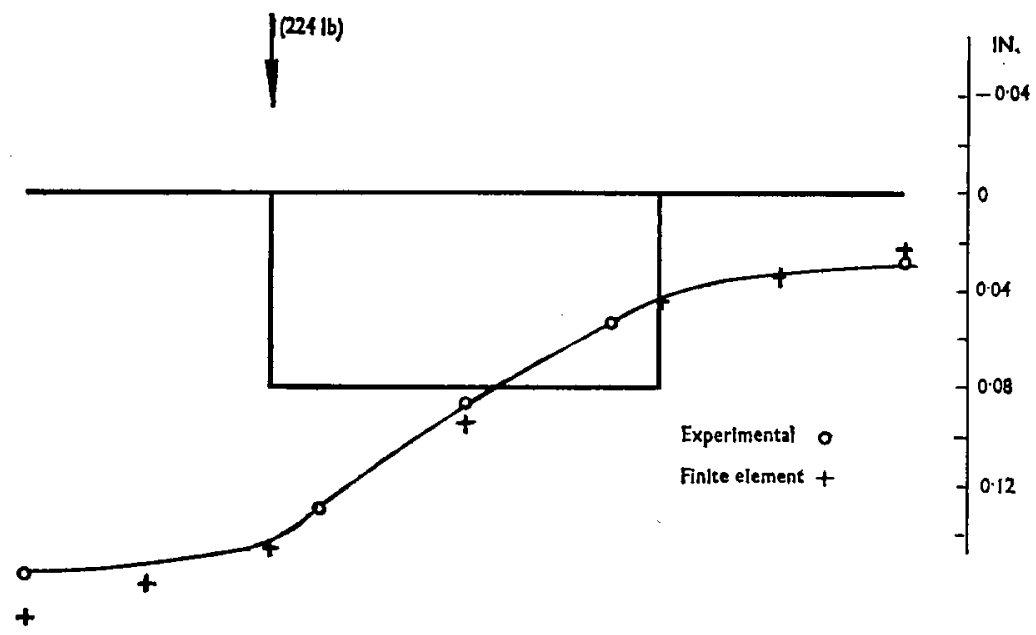

Fig. 16. Deflexions at mid span 
DISCUSSION

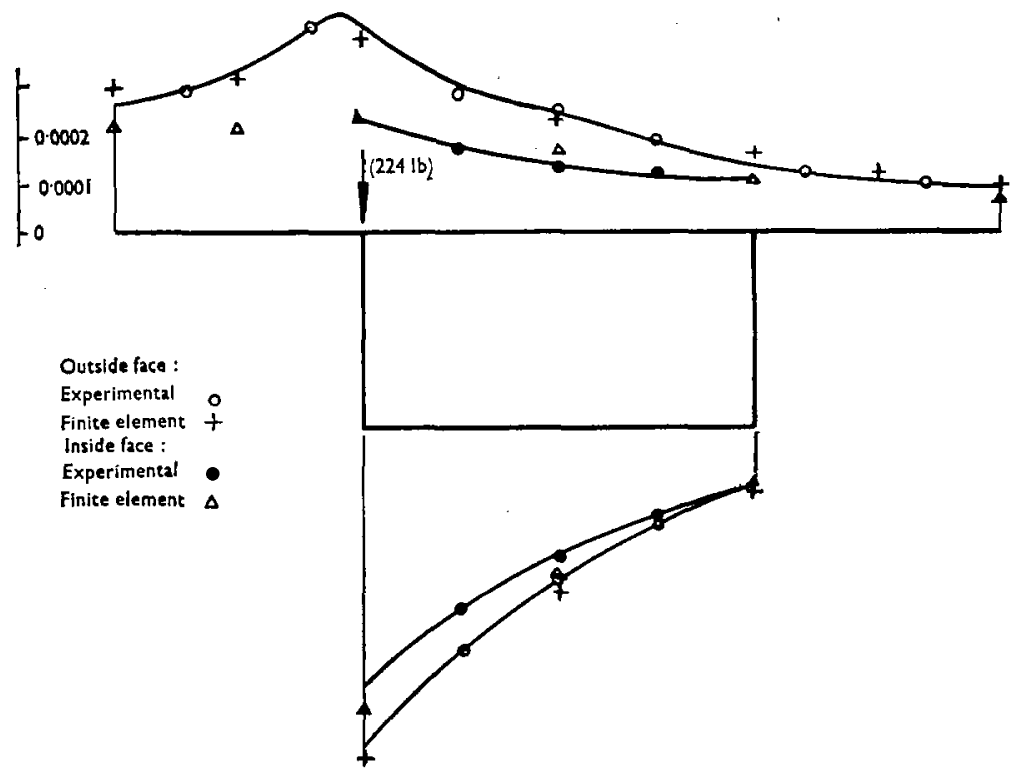

Fig. 17. Longitudinal strain on line B

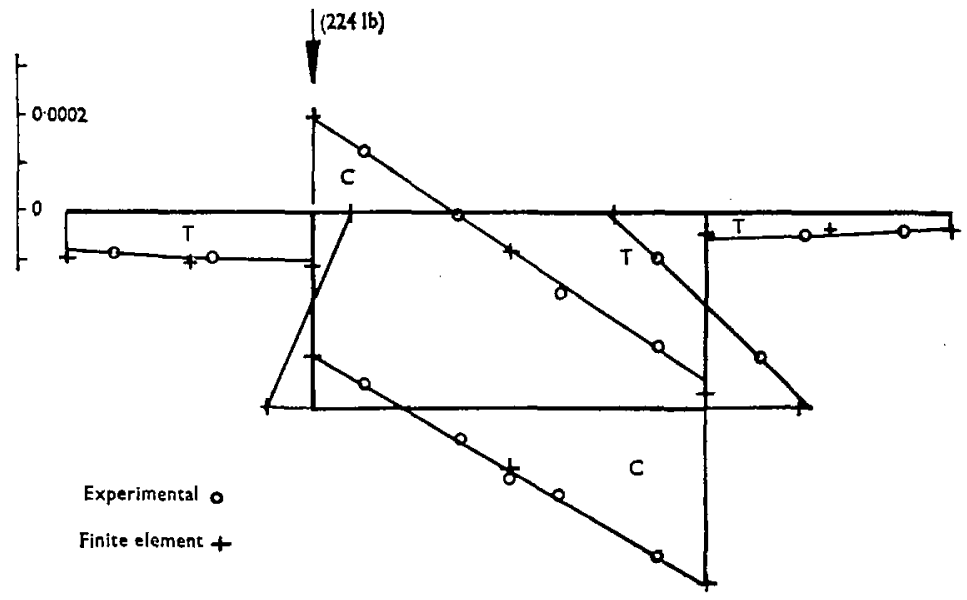

Fig. 18. Transverse strain on outside face 
program for a structure such as a multi-store, multi-bay frame with the flexural and in-plane stiffnesses of walls and floors included in the analysis. We believe that further advances in computer storage technology and solution methods will have to precede the practical analysis of these systems.

\section{Dr W. M. Jenkins and Mr G. C. de Jesus, University of Bradford}

The Paper is a significant contribution to computer aided analysis of complete structures comprising diffcrent types of structural element.

48. Referring to the flow diagram (Fig. 7) and to Table 1, it is noted that the computation of the displacement vector $\boldsymbol{X}$, element forces $\boldsymbol{P}_{\mathrm{f}}, \boldsymbol{P}_{\mathrm{O}}$ and $\boldsymbol{P}_{1}$ and load vector $L_{\mathrm{R}}$, requires the provision of storage sufficient to accommodate eight matrices of relatively large dimensions. Even if the maximum amount of overwriting is carried out in the subsequent calculations, the initial requirements are large. Matrices $K_{0}, K_{\mathrm{t}}$ and $K_{\mathrm{f}}$ cach requires $N \times N$ locations ( $\left.\$ 12\right) ; k_{\mathrm{t}} A_{\mathrm{f}}, \boldsymbol{k}_{0} A_{0}$ and $\boldsymbol{k}_{1} A_{1}$ each requires a variable number of locations depending on the number of each type of element, and the total of these three could easily exceed $N \times N$. Thus it is clear that the procedure described can be executed only on a large machine, even for structures with relatively small numbers of degrees of freedom.

49. The necessity of providing a large store is a serious restriction in view of the present lack of really large capacity machines and it is desirable to formulate the solution using techniques which make more modest demands on computer storage.

50. We have found that the method described below meets this requirement.

(a) Basic data is read including indications of the method of connexion of the elements and the geometrical and structural properties of the elements. If the number of different types of element is not large, the individual stiffnesses need be calculated only once and stored. However, if this requires a substantial amount of storage, an alternative is to compute the element stiffinesses as they are required in the process of stiffness matrix assembly.

(b) The structure stiffness matrix is assembled not by the triple matrix multiplication used by the Authors, but by a process of direct addition or merging of the element stiffnesses. Whilst this process is perhaps not as elegant as the matrix operation, the final result is of course identical and storage requirements arc minimized. Moreover, programming has not been found to be unduly complicated. One-half band of the symmetric stiffness matrix is stored. Thus, if the semi-band width is $B$, the storage required is $B \times N$ locations.

(c) The system of equilibrium equations is then solved for each loading vector required. The solution process is programmed so that only a portion of it is repeated for each new loading case. The applied load vector is read in one set at a time. The calculated displacements are of course referenced to the structure axes but these can easily be converted to element nodal displacements by suitable transformations.

(d) Element forces are calculated from the element stiffnesses and nodal displacements. As in the formation of the overall stiffness matrix, the element matrices can be re-computed as they are required. The computation of the reactions presents no difficulty when this stage has been reached.

51. The overall computing time for the procedure outlined may be somewhat longer than for the Authors' program, on the other hand the economy achieved in storage requirements enables the solution of structures of comparable complexity on medium or even small sized computers. It is estimated that, for the last example solved by the Authors, the storage requirement need be only 10000 locations. Even this is based on the assumption that the sheeting has been divided into twenty-four 
elements. This requirement can be met by several medium sized machines using the fast store alone.

52. The correlation between the theoretical and experimental results quoted for the examples in the Paper is remarkably good. However, in the case of the shed, the Authors did not offer any explanation of the tendency of the theoretical results to diverge away from the experimental values as the number of elements was increased.

\section{Mr J. B. Bellamy, Research Student, University College of Cardiff}

The Authors are to be congratulated on their important contribution to the study of the influence of plate elements on the behaviour of a skeletal frame. The correlation between the theoretical and experimental results that they present, particularly for the pitched roof portal shed, is most interesting. In the main the Authors have obtained better theoretical predictions of the behaviour of the shed by representing the cladding by a coarse finite element mesh. This is contrary to the experience of many investigators into finite element techniques who have found that the accuracy of their results for a particular problem is proportional to the number of elements that they use. Seeing that their theoretical results for deflexions are usually underestimates and their bending moments overestimates of the behaviour of the shed, would the Authors agree that this divergence is due to less rigidity in the connexions of the model than that assumed in their analysis and, if so, have they considered using special elements in their program to cope with such movements? Such special elements could be of two types: firstly to represent the connexions between the cladding and the frame; secondly to represent the lack of a full connexion between the various sheets comprising the cladding. The latter type of special element would nced to have little or no resistance to certain types of distortion so that larger deflexions could occur. This technique is inherent in the difference between analysing a frame as being rigidly jointed or as having rotationally free joints.

54. My second comment concerns the problem caused by the absence of any terms for the in-plane rotation of the corners of the elements in the element stiffness matrices. This is a well known defect of the plate element that the Authors have adopted. Their solution is to insert two diagonal prismatic elements "between the opposite corners of any plate whose in-plane rotational stillness is considered to be important'. Perhaps the Authors would like to outline their criterion of importance. Furthermore, are the Authors sure that they are not including the stiffness of the plate element twice in such a case. that is in effect adding additional members to the structure they are analysing, which do not exist in the real structure?

55. I was very disappointed in the size of problem that the Authors' computer program can tackle and I am rather surprised that they did not attempt to use magnetic tape storage from the start of their research. I am currently undertaking a project to study the stiffening effect of the cladding upon the behaviour of a space frame, with the ultimate intention of showing that the upper layer of a double layer skelctal space grid can be rcplaced by a slightly stiffened cladding. This research is the natural extension of previous work by Evans ${ }^{9}$ and myself, ${ }^{10}$ and involves a theorctical approach similar to that used by the Authors of the present Paper. A computer program, using a tridiagonalization scheme of stiffness matrix handling and magnetic tape storage, is at present at an advanced stage of devclopment. It is a little early to say exactly but it is expected that the program will be able to analyse structures having over 2000 degrees of freedom, on an Allas computer.

56. Finally, I would like to take up the Authors' claim that their Paper is the first attempt to analyse a complete structure in the field of civil engineering. The finite element technique is now considered a basic tool by structural research engineers and many investigators both in Europe and the USA, as well as in this country are using mixed element methods of analysis. In particular, have the Authors considered the work of Argyris ${ }^{11}$ and Zienkiewicz, ${ }^{12}$ both of whom use mixed element methods of analysis, or are they setting unusually narrow limits to the field of civil engineering? 
Professor I. C. Medland and Mr C. C. Yao, University of Toronto

We are interested in the work of Dr Majid and Mr Williamson since similar thoughts to those expressed in the introduction to the Paper prompted us to proceed with a more limited investigation of a similar nature. ${ }^{13}$

58. The Authors have shown a method whose power justifies the claim that it is a method of analysis of complete structures. In practice, however, difficulties of computer capacity in any non-regular or large structure would severcly limit the number of elements into which the plates, say, could be subdivided. This would mean that internal forces in such members would presumably have to be handled by further analysis with separate storage.

59. The results given, however, indicate that a relatively coarse subdivision of plate members yiclds very close results in terms of overall effects. This aspect deserves considerable attention since, in our opinion, the practical usefulness of such comprehensive analyses lies principally in their ability to furnish further information whereby three dimensional effects (extra stiffness, etc.) can be related to the basic plane frame analyses. Our interest in the subject was directed towards the additional in-plane sway stiffness added to one plane frame by its parallel neighbours connected by floor slabs only.

60. In order to gain some idea of the importance of this extra stiffness a preliminary analysis was carried out on table structures (Fig. 19) similar to those described

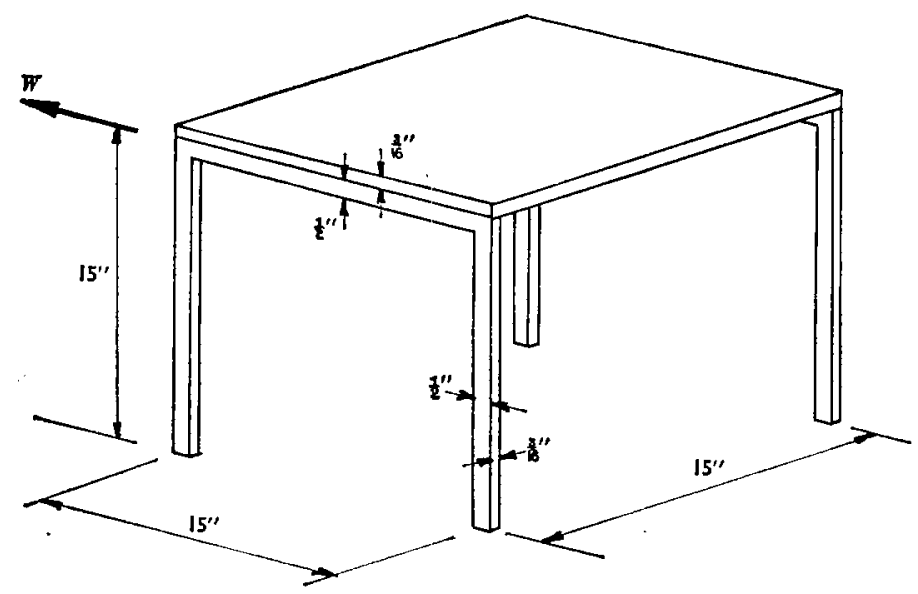

Fig. 19

by the Authors in $\S \S 28-32$. The effect of the slab, apart from its basic control of the plan geometry, was considered to be simply an addition to the beam bending stiffness. This addition was originally estimated by considering a standard width of slab (6x thickness) to act integrally with the beam. When test results were examined it was readily seen that the theory was very sensitive to the width taken and that this width was obviously some function of other geometrical proportions, principally the bent spacing, to frame span ratio. Thus the Authors' approach, with as few as nine elements in the slab, provides a method of readily assessing such a width for practical use if the above approach is made.

61. Since the model tests recorded ${ }^{13}$ were of similar size to those of the Authors, it is of interest to note that the perspex structure shown in Fig. 19 showed 


\section{DISCUSSION}

$40 \%$ increase in stiffness (against the one load $W$ ) over the bare frame. A similar sized model in steel gave $30 \%$ increase. Part of our investigation was to assess the magnitude of these increases approximately in order to determine whether an approach such as the Authors' was worth while. The above figures seem to indicate that it is. However, if applied to a pair of frames comprising structural members (beams 14WF30, columns 10WF49) linked by a 4 in. concrete slab, the theories yielded only a $5 \%$ increase. No tests were done on such frames. In our opinion this still indicates a worthwhile contribution particularly from a stability aspect since the sway instability load is extremely sensitive to this sway stiffness term. The relatively low torsional and minor axis properties for an I-section, as opposed to a rectangular section, account for this smaller increase.

\section{Dr J. Khanna, Swan Wooster Engineering, Vancouver, Canada}

The Authors presented interesting examples of the computer analysis of space structures composed of prismatic members, and rectangular plate elements, and provided experimental verification of the computer results.

63. They claimed that the attempt in the Paper to analyse complete structures using the above elements was 'the first of its kind in the field of civil engineering', however, my firm has been using a space frame program incorporating rectangular finite elements for over two years.

64. I feel that the Authors should have clearly stated that their Paper lay within the extensive domain of the finite element method. Such a characterization would have reduced the necessity of elaborate descriptions, contained in the Paper, of standard matrix structural analysis procedures. Also, it would have been clear that other one, two, and three dimensional finite elements could be used with equal ease in the idealization of complete structures. As an example, the Swan Wooster space structure program, in its present form, allows the use of non-prismatic members, elastic translational and rotational springs, triangular plate elements and rectangular shell elements (incorporating in-plane and out-of-plane forces) in addition to the elements used by the Authors.

65. It is also worth noting that although the Authors confined themselves to linear static analysis, the analysis is easily extended to a study of the elastic dynamic behaviour of complete structures. In this case the stiffness matrix of the structure, which is obtained during the static analysis, could be used to obtain the natural frequencies and mode-shapes of the structure by solving the usual eigenvalue problem. ${ }^{14}$ Then, a dynamic force could be applicd to the structure, ${ }^{14}$ and a matrix step-by-step integration procedure used to obtain a time history of the dynamic response of the structure. For example, the Swan Wooster program allows the dynamic analysis of complete structures as options in the same run as for the static analysis.

\section{Mr M. A. Khan, Civil Engineering Department, University College London}

The Authors should be congratulated for developing a finite element approach for the analysis of complete slab-frame and slab-frame-shcar wall structures.

67. It will be relevant to describe here briefly the theoretical and experimental work on the subject of slab-frame interaction which I carried out, at University College London, using a different theoretical approach. The studies were mainly confined to T-beam action in single and continuous panel monolithic slab-beams, though the case of the combined slab-frames under the action of both transverse and lateral loads was also investigated using the method of flexibility coefficients. In this method the columns were released from the slab-beams at the intersection point with the beams. The load-displacement coefficient of the slab-beams for the transverse loads was obtained by solving the plate equations and the boundary equations on the Atlas computer using the finite difference method. Both full and partial composite action between slab-beams were considered. 
68. The flexibility coefficients of the slab-beams were obtained by applying unit external moments and external axial forces at the corners of slab-beams. The conditions of compatibility for the rotations and translations at the junctions of the columns and the slab-beams were then applied by making use of the load-displacements and flexibility coefficients of the slab-beams and the columns, and the slabframe restored to its original form.

69. A series of tests on perspex slab-frame models resembling a table have shown close agreement with the theory. ${ }^{15}$

70. - It was found that for the case of a slab-frame under transverse uniformly distributed beam loads the beam moments both at mid-span and at the supports of beams were lower by over $50 \%$ compared to a simple bare frame of identical stiffness and under the same loading. The percentage of reductions in the beam moments varied with the stiffness of the columns and the relative stiffness of beam to slab. The maximum moments in the columns of the composite slab-frame were similarly lower than those in bare frame columns by over $50 \%$.

71. For non-symmetric wind loads assumed to act at the joints of the columns and beams, the beam support moments were reduced by over $35 \%$ compared to an identical bare frame under the same loading for certain practical stiffnesses of the columns and for certain practical stiffness ratios of beam to slab. While detailed comparisons with the Authors' results are not made here, Dr Majid and $\mathrm{Mr}$ Williamson's results for the case of lateral loads which show an increase of $28 \%$ in the stiffness of the bare frame when slab is present, in general suggest the same order of reduction as obtained by the flexibility coefficients approach.

72. I would like the Authors to answer the following questions.

(a) Were the planar forces in the slab and beams measured experimentally and their magnitude and distribution compared with the theoretical planar forces in the slab and beams arising due to T-beam action in slab, and if so what kind of agreement was obtained?

(b) It is seen from Fig. 8 that the experimental load-deflexion graph agrees more closely with the nine elements analysis than with the twenty-five elements, specially for the lower load range. Yet the Authors conclude that if plates are sub-divided into smaller elements accurate results would be obtained. This point is not clear.

(c) From the experimental perspex table with a plate size of $18 \mathrm{in.} \mathrm{square}$ and $\frac{1}{16}$ in. thick, it is seen that a high span/thickness ratio $(=288)$ is obtained. Due to the in-plane forces the plate along with the edge beams would be subjected to bending. Do the plate deflexions for the load range shown exceed the plate thickness of $\frac{1}{16} \mathrm{in}$. and if so does the thin plate action give rise to tensile membrane forces in plate and to non-linear deflexions of the plate?

(d) Finally in the experiments on the table with a top plate loaded transversely, was the agreement with theory as close as for the case of in-plane loads?

\section{Dr Majid and Mr Williamson}

The main points raised by the contributors are:

(a) Can the finite element approach be applied to civil engineering problems and how efficient is such an approach?

(b) Does the subdivision of plate elements lead to an improvement in the overall analysis of the structure as a whole?

(c) How does one treat the in-plane rotational stiffness of the plate component of a structure?

(d) How does one overcome the difficulties imposed by the limits of present-day computers? 


\section{DISCUSSION}

74. In reply to the first two points the Authors wish to make the following two distinctions.

75. The distinction between matrix-displacement method and finite element approach. While the matrix displacement method is an accurate scientific tool with which to analyse structures, discrete or continuum, the finite element approach is an approximate physical representation of two and three dimensional elements. The approximations are justifiably made so that stiffness matrices can be derived for use in the matrix method and the penalty paid for the approximation is some loss of accuracy.

76. The distinction between the analysis of aircraft and civil engineering structures. The two fields naturally overlap to a limited degree. However, particularly in the analysis of aircraft structures the plate components, particularly under in-plane (skin) forces, play a preponderant part while the bare frame consists of light and slender members neatly connected to cach other and to the plates at common nodes. Civil engineering structures, by their functional nature, consist of many irregularities, in which the bare frame, of deep and stocky as well as light and slender members, has a significant part to play. The arch and the steel cables of the bridge shown in Figs 20 and 21 form a good example.

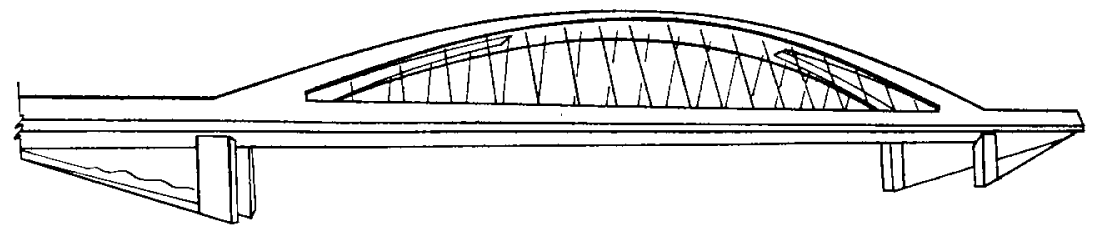

Fig. 20

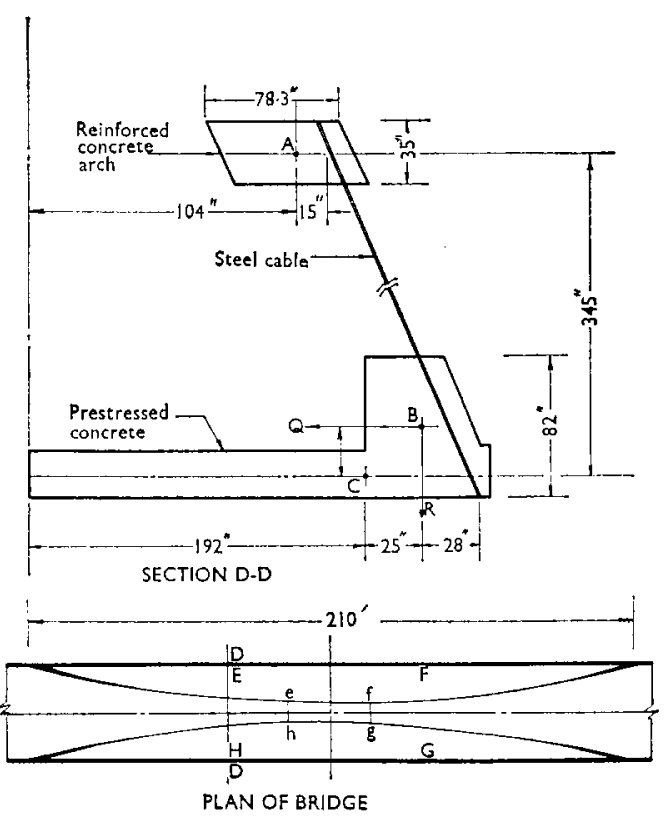

Fig. 21 
77. For the above reasons, the finite element technique of plate analysis has been developed and applied in the aircraft industry. The work of Argyris et al. and Melosh are fine examples. The plate element forces, being significant, are looked into in detail and subdivision of the plates naturally leads to an improvement in their representation. However, subdivision incrcases the size of all the matrices, and, particularly that of the overall stifiness matrix that has to be inverted. The result is some loss of accuracy and thus after an optimum mesh size, which is dependent on the type of the structure, any further subdivision would lead to diverging results. This characteristic of the finite element technique has also led to the development of various improved element stiffness matrices. The process of structural analysis continues by simple addition of the overall stiffness matrices of the various idealized components. This is very useful and convenient in the analysis of aircraft structures.

78. Unlike this 'narrow limit' imposed by Mr Bellamy, many civil engineering structures cannot be simplified to idealized uniform elcments neatly meeting at their common nodes. Repeated subdivision of the plates may improve the analysis of these plates but we showed that the effect of plate subdivision has little influence on the overall accuracy of the structure (\$\$ 29,31 and Tables 2 and 3$)$. It was the overall understanding of the structures that led us to refrain from giving all our attention to the plates and instead concentrate on the rest of the structure. This was reflected in the Paper where the results presented were all concerned with the bare frame part of the structures analysed-results of what was happening in the plates were not mentioned. This was because a detailed study of the plates would only have verified the accuracy, or otherwise, of the various plate stillness matrices developed by Argyris, ${ }^{5}$ Mclosh ${ }^{8}$ and Zienkiewicz and Cheung, ${ }^{7}$ and used in our programs. In the case of the table with a top plate loaded transversely (\$32), it was noticed that a finer mesh of 24 elements was required to give good agreement with experimental results obtained for the plate element forces. This confirms our conclusions.

79. A significant improvement in the analysis of civil engincering structures is achieved by including the physical features of these structures. For this reason instead of pursuing the development of the plate element stiffness matrix $k$, we concentrated on the displacement transformation matrices $A$ used in the analysis. For instance the railway bridge, analysed for Messrs Husband \& Co. shown in Figs 20 and 21 is made of steel, prestressed concrete and reinforced concrete components. The material propertics such as cracking, etc. of these are so different from the idealized ones that the very recent process, of finite element as applied to aircraft structures, is inadequate to cope with them. Moreover the alteration needed is not so much an improved $\boldsymbol{k}$ but an improved $\boldsymbol{A}$ matrix. Fig. 21 shows that at a particular cross section of the bridge the steel cable is 15 in. from point $A$. the centre of the arch, which is a node in the structure. If point B, the centroid of the longitudinal beam, is taken as another node and the cable is assumed to be connected to $A$ and $B$, while the deck plate is also assumed to be meeting the member at $B$, then this erroneous simplification yields results that bear no resemblance to the actual state. The gross alteration of the geometry of the structure and the $T$ beam effect, rightly stressed by $\mathrm{Mr}$ Khan, would have been but two of the total casualties. The conditions at every node were different but none, except within the deck, would have becn idealized and Messrs Husband \& Co. were particularly concerned with these irregularities. Using the proposed method, it was decided to insert the nodes at $A$ and $C$ and introduce the offsets $q_{\mathrm{c}}$ and $r_{\mathrm{c}}$ of 34 in. and 25 in. respectively for the longitudinal beam togcther with appropriate offsets for the cable. These offsets were inscrted into the displacement transformation matrix of these members. The matrix $A_{t}$ of equation 17 was thus altered to become :

$$
A_{t}=U_{1} V_{I} \quad \text {. . . . . . . . . }
$$


In equation $18 V_{f}$ is the matrix of direction cosines ${ }^{4}$ while,

$$
\begin{aligned}
& \text { at joint } J
\end{aligned}
$$

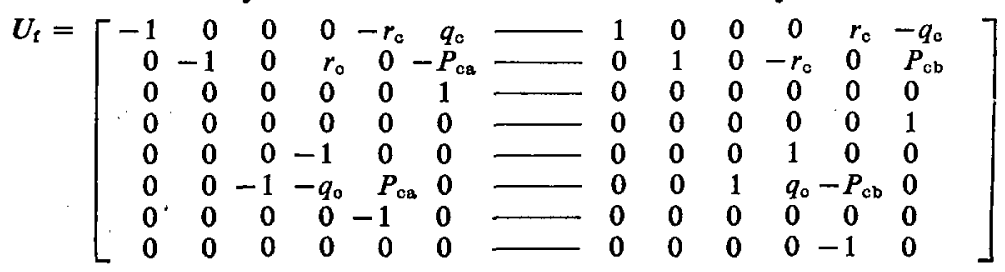

Matrix $U_{\mathrm{f}}$ presents to the analysis the special features of the bulky prismatic members. The offsets $r_{0}$ and $q_{0}$ are those of the centroidal $R$ and $Q$ axes of a member from the node to which it is connected. These also take care of the $\mathrm{T}$ beam effect of the monolithic plate/member connexion. $P_{\mathrm{og}}$ and $P_{\mathrm{ab}}$ are the lengths of the gusset plates, massive haunches or welded parts (in the case of the bridge note the bulged ends of the arches where they meet the deck plate). In addition the computer program introduces the offsets $q_{\mathrm{s}}$ and $r_{\mathrm{a}}$ of the shzar centre from the centroid, as well as the product moment of area $I_{\mathrm{ar}}$ of unsymmetrical sections, to the stiffness matrix of the prismatic members. For details of this, reference was made to a previous paper by Jennings and Majid. ${ }^{4}$ The electrofilter precipitator casing of Fig. 22 is another civil engineering structure which was originally designed by Messrs Lodge Cottrell to the present BS 449. Here again the structure contains a vast number of deep joists shown as full lines in the figure. Any simplification by assuming that the centre lines of these members coincide with the plane of the plates would naturally lead to miscalculation of the stresses.

80. An interesting feature of civil engineering structures as distinct from aircraft structures, was pointed out by Professor Medland and Mr Yao. The figures they quoted show how the cladding effect of various materials differ a great deal. We look forward with interest to their experimental results for the table with concrete top because this will bring new features of the actual structure to light where again the existing simple element stiffness matrices would not be of much value. We are considering the modification of the various displacement transformation matrices to cope with the problems of cracking of concrete elements but admit the difficulties of such a task. Under these circumstances we confirm that the application of the matrix displacement method to the wider fields of civil engineering is still in its first stage. As for the facilities available at Dr Khanna's organization, we would have been very much interested in a reference to his published work on the subject so that we would have been in a better position to comment.

81. To confirm that greater accuracy of the overall analysis of the structure does not follow naturally from the mere subdivision of the plate elements nor from the use of improved stiffness matrices, we used the early and crude out-of-plane stiffness matrix of Melosh ${ }^{6}$ (1961) and the much refined recent matrix of Zienkiewicz (1964) and found that for the same number of plate elements, while the matrices gave different results in the plate portion of the structures, their influence on the prismatic member forces and joint displacements were very much the same. Subdividing the plates, using either matrix, did not alter the situation significantly. We presented our results not to throw doubts on the process of subdivision or the accuracy of matrices developed by other authors but rather to confirm that most of the results were indeed in agreement with the experimental values. It should be repeated that, in the particular case of the semi full scale shed, the stiffness of the corrugated sheeting was obtained experimentally $y^{2.3}$ to be used later as that of plane sheets in the analysis. This idealization would naturally bring about some discrepancies with the actual 


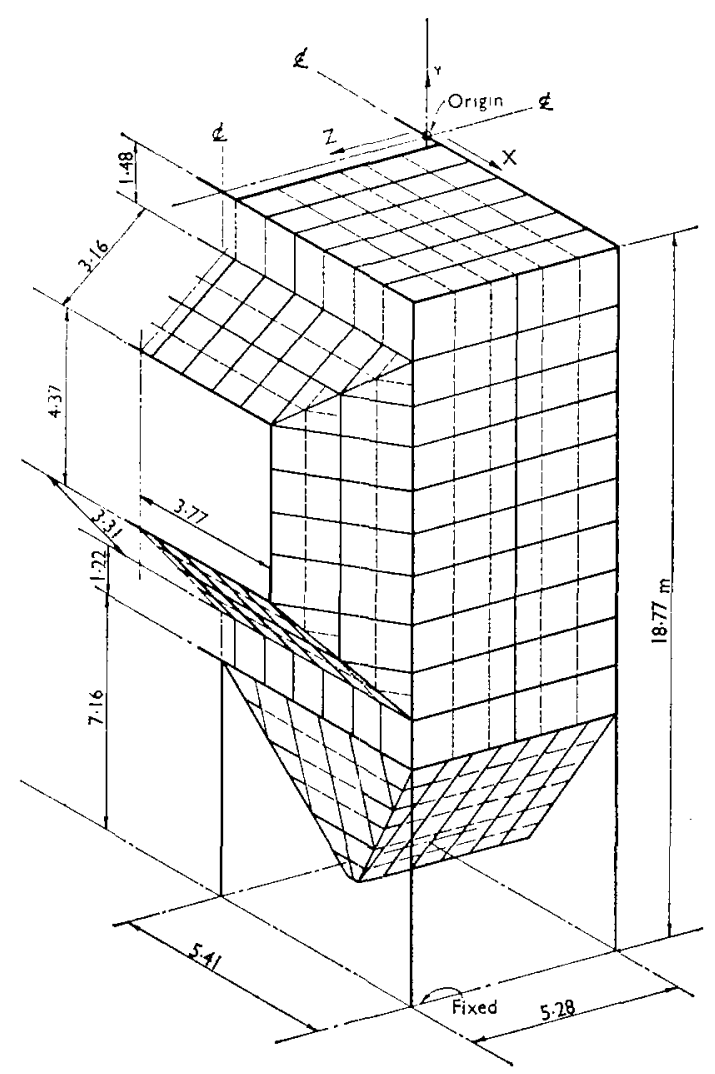

Fig. 22

behaviour of the plates. Nevertheless the results given in Tables 2 and 3, for the eaves of the prismatic members agree astonishingly well with the experimental results. Once again subdivision of the plates from 9 to 18 elements caused only minor differences.

82. Another weakness of finite element approach is its incapacity to deal with the in-plane rotation of coplanar elements. This is because the rotational stiffness of a plate is unknown. This defect as Professor Sawko and Dr Cope pointed out is not so important within the plate elements themselves because of their high rotational stiffness. It does, however, bring about two problems. First, a degree of freedom about $R$ axis at a node to which only plates sustaining membrane stress are connected results in a singular overall stiffness matrix. On the other hand suppressing it prevents the out-of-plane rotations of non coplanar elements as well as those of prismatic members surrounding or supporting these membrane elements.

83. The criterion of importance is thus decided by the nature of the structure and the error that is introduced in the problem. For instance in the case of the table of Fig. 19 a couple, applied in the plane of the slab, twists the columns and suppressing the in-plane rotation of the nodes within the slab, results in an erroneous calculation of the columns' torque. The structure of Fig. 22 is an example where this problem 
does not even occur since at each node of a plate"there is at least one prismatic member to resist the rotation. Each joint can therefore have six degrees of freedom. The bridge of Figs 20 and 21 is an example where the manner of loading dictates whether or not in-plane rotation is significant. When one half or the whole of the deck is loaded, the rotation of the bridge about the vertical $Y$ axis is of no importance. On the other hand skew symmetric loading of opposite quarters, marked $F$ and $H$ in Fig. 21, leads to the rotation of the top shell efgh. Suppressing the in-plane rotation of the top shell would restrict the twisting of the arches.

84. The suggestion made by Professor Sawko and Dr Cope to derive a new stiffness matrix using deflexion functions compatible with the normal displacement of the flexural function is one way of treating this problem since this is an improvement on the stiffness matrices ${ }^{5,6,7}$ which we used. However the difficulty of selecting a reasonable and conforming displacement function remains to be solved. Past experience of research workers shows that each displacement function used results in a stiffness matrix of limited application.

85. For the time being there is more than one way of coping with this difficulty. One method is to insert two diagonal members across a plate. The propertics of these members are selected such that they only offer bending stifiness in the plane of the plates. All the other properties of these members, such as direct, torsional and bending stiflness out of the plane of the plates are set to zero. Thus these members do not add additional stiffness to the structure. Another method is to have members that run along the subdivision lines of the plates. For instance in the case of the table of Fig. 19 these members would be selected with such a bending stiffness that by their rotation they would transmit all the applied torque to the columns. Any error introduced to the stillness of the plate can be detected by another analysis of the table with these members removed. Introducing rotational springs with one end connected to a node and the other end fixed is another way of treating this problem. In the case of the bridge of Figs 20 and 21 , the in-plane rotational degree of freedom at nodes within the top shell efgh were suppressed for the symmetric and antisymmctric loading cases. For the case of the skew symmetric loading mentioned above, the shell eligh was replaced by equivalent members. This enables the calculation of the torque in the arch.

86. The time taken for the structures analysed was exceptionally small. The largest structure that was given in the Paper was the semi full scale shed having 41 joints with 191 degrees of freedom, 60 members and 18 plates. As stated the total time for four loading cases was only one minute (10 000 machine instructions). It is to be remembered that this time also included the reading and compiling of the program as well as the special sparse matrix routines used in the analysis. For a general structure, taking all the irregularitics of the frame into consideration, the approach suggested by $\mathrm{Dr}$ Jenkins and $\mathrm{Mr}$ de Jesus is very difficult particularly when the matrices are stored and operated upon in their sparse form. Furthermore, because of the sparse matrix storage method, the figures suggested by $\mathrm{Dr}$ Jenkins and $\mathrm{Mr}$ de Jesus for the size of the various matrices are grossly overestimated.

87. Naturally, reducing the capacity of a program to deal only with simplified structures or writing a program that analyses one or a few structures of a given geometry as suggested by Professor Sawko and Dr Cope will result in economy in the storage requirements. This is mainly because the programs make use of the specific features of the structure to reduce storage requirements. Indeed the structures themselves being 'very elementary' would be less demanding. However this approach means writing a new program for every structure, which not only avoids the issue of producing a general program once and for all, but indeed a great deal of time is consumed in the preparation of these separate programs.

88. In the concluding remarks to the Paper we intended to pose the question about storage requirements rather than answer it since that in itself would have required a separate paper. Unlike Professor Sawko, Dr Cope, Dr Jenkins and Mr de Jesus, 
we believe that the present-day computers have all the storage facilities for the analysis of any structure irrespective of its size or geometrical irregularities. Since this Paper was written Majid and Anderson ${ }^{16}$ have presented a paper to The Structural Engineer for the analysis of complex structures without the use of magnetic tape storage facilities. In this paper the linear as well as non-linear analysis of a sixty storey five bay plane frame with 660 joints and 1980 degrees of freedom is given as an example. Nevertheless as the number of degrees of freedom increases sooner or later magnetic tapes become necessary. We agree with Mr Bellamy's approach of using a tridiagonalization scheme particularly because this scheme is well documented. ${ }^{17}$ Since the present Paper was written, we have produced such a method of analysis for complete structures, using a tridiagonalization scheme. ${ }^{B}$ A specification for the computer program is now available ${ }^{18}$ and a paper is being prepared for the work. The main improvement to the tridiagonalization scheme is the introduction of sparse matrix technique. Using this scheme the bridge of Figs 20 and 21 was analysed. Making use of the symmetry of the structure only a quarter of the bridge was analysed. This consisted of 120 joints with 555 degrees of freedom, 50 members and 64 plate elements. A quarter of the precipitator of Fig. 22 was also analysed. This consisted of 390 joints with 2064 degrees of freedom, 517 members and 296 plates. Because of the large number of members in the structure only minor subdivision of the plate was required. These are shown as dotted lines in Fig. 22. It appcars that Mr Bellamy is also intending to take a course similar to ours and we look forward with interest for him to complete his work to compare results.

89. The remarks made by $\mathrm{Mr}$ Khan about the agreement between his results and ours are encouraging. Since this Paper was written our computer program has been used by B. Hayes ${ }^{19}$ for an extensive study of the $\mathrm{T}$ beam action in monolithic slabbeams and comparison has been made between this approach and that of using finite difference. Some of the models tested are shown in Fig. 23. The slabs were

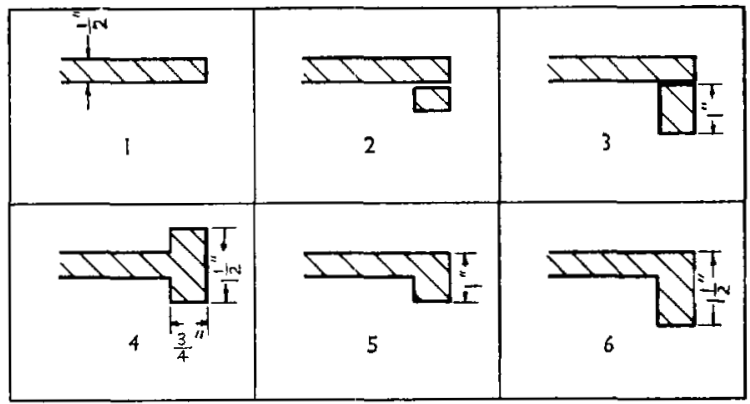

Fig. 23

$18 \frac{3}{4}$ in. square and made from $\frac{1}{2}$ in. perspex sheet. The beams were $\frac{3}{3}$ in. wide and $\frac{1}{2}$ in., 1 in. or $1 \frac{1}{2}$ in. deep with the panels subject to UD load. In Table 4 the results obtained by the method are compared with those obtained by experiment and by finite difference. For the first four edge beam conditions no $\mathrm{T}$ beam action is present and a bending only analysis with zero of isets has been used. It is observed that the two methods are in agreement with each other and with the experiments.

90. In slabs 5 and $6, T$ beam action is prescnt and analyses including and neglecting in-plane stresses have been carried out using a $6 \times 6$ mesh for the panel. The results in Table 4 show that neglecting $\mathrm{T}$ beam action both methods give erroneous results. On the other hand including the $T$ beam action improves the results to bring them 
Table 4. Correlation between perspex model slab results and analyses by finite difference and finite element methods

\begin{tabular}{c|c|c|c|c|c}
\hline \multirow{2}{*}{$\begin{array}{c}\text { Model } \\
\text { slab no. }\end{array}$} & \multicolumn{5}{|c|}{ Central deflexions in./lb/s } \\
\cline { 2 - 4 } & Exp. & $\begin{array}{c}\text { Finite } \\
\text { diff. }\end{array}$ & $\begin{array}{c}\text { Finite } \\
\text { diff. }\end{array}$ & $\begin{array}{c}\text { Finite } \\
\text { element }\end{array}$ & $\begin{array}{c}\text { Finite } \\
\text { element }\end{array}$ \\
\cline { 2 - 4 } & 0.486 & 0.514 & 1.06 & 0.487 & Exp. \\
\hline 1 & 0.444 & 0.468 & 1.05 & 0.442 & 1.00 \\
\hline 2 & 0.294 & 0.301 & 1.02 & 0.286 & 1.00 \\
\hline 3 & 0.174 & 0.177 & 1.02 & 0.169 & 0.97 \\
\hline 5 & 0.255 & $\begin{array}{c}0.236 \\
(0.292)\end{array}$ & $\begin{array}{c}0.93 \\
(1.15)\end{array}$ & $\begin{array}{c}0.236 \\
(0.278)\end{array}$ & $\begin{array}{c}0.97 \\
(1.09)\end{array}$ \\
\hline 6 & 0.143 & $\begin{array}{c}0.135 \\
(0.184)\end{array}$ & $\begin{array}{c}0.94 \\
(1.29)\end{array}$ & $\begin{array}{c}0.131 \\
(0.177)\end{array}$ & $\begin{array}{c}0.92 \\
(1.24)\end{array}$ \\
\hline
\end{tabular}

Figures in parentheses refer to analyses for slabs 5 and 6 neglecting $T$ beam action.

within $92 \%$ of the experimental values. Similar correlation has been obtained for centre line stresses for uniform loading and stresses and deflexions for central point loading.

91. The answers to Mr Khan's other questions are given earlier except that it should be mentioned that the proposed method only deals with linear analysis using small deflexion theory. Non-linearity due to large deflexions is not considered. For this reason better agreement with experimental results was obtained by using thicker plates.

\section{References}

9. Evans H. R. The analysis of folded plate structures. PhD Thesis, 1967, University of Wales, Swansea.

10. Bellamy J. B. The elastic stability of space frames. MSc Thesis, 1967, University of Wales, Swansea.

11. ARgyris J. H. Continua and discontinua. Proceedings of conference on matrix methods in structural mechanics, 1965, Wright-Patterson Air Force Base, Ohio, USA.

12. ZIENKIEWICZ O. C. The finite element method in structural and continuum mechanics. McGraw-Hill, 1967.

13. Yao C. C. Integral action in structural frames. MASc Thesis, 1967, University of Toronto.

14. FINN W. D. et al. Dynamic response of earth dams. Proceedings of the third symposium on earthquake engineering, 1966, Roorkee, India, 315-325.

15. Khan M. A. Elastic interaction between floors and frames. Thesis to be submitted shortly to the University of London.

16. MAJid K. I. and ANDERSON D. The computer analysis of large multi-storey framed structures. Struct. Engr. To be published. 
17. RuBINSTEIN M. F. Matrix computer analysis of structures. 1966, Prentice-Hall, Inc.

18. MAJID K. I. Specification for analysis of large complete structures using sparse matrix storage system and tridiagonal banded sub-matrix operation. Department of Civil Engineering, University of Aston in Birmingham.

19. HAYEs B. PhD Thesis, 1968, University of Manchester. 